EPJ Web of Conferences 59, 17014 (2013)

DOI: $10.1051 /$ epjconf/20135917014

(C) Owned by the authors, published by EDP Sciences, 2013

\title{
Longitudinal proton probing of ultrafast and high-contrast laser-solid interactions
}

\author{
B. Albertazzi ${ }^{1,2}$, P. Antici ${ }^{3}$, J. Bocker ${ }^{4}$, M. Borghesi ${ }^{5}$, S. Chen ${ }^{6}$, V. Dervieux ${ }^{1}$, \\ E. d'Humières ${ }^{7}$, L. Lancia ${ }^{3}$, M. Nakatsutsumi ${ }^{1}$, R. Shepherd ${ }^{6}$, \\ L. Romagnagni ${ }^{1}$, Y. Sentoku ${ }^{8}$, M. Swantusch ${ }^{4}$, O. Willi ${ }^{4}$, H. Pépin ${ }^{2}$ \\ and J. Fuchs ${ }^{1}$
}

1 LULI, École Polytechnique, CNRS, CEA, UPMC, 91128 Palaiseau, France

2 INRS-EMT, Varennes, Québec, Canada

3 Dipartimento di Energetica, Universita di Roma 1 "La Sapienza", Roma, Italy

4 Institut für Laser-und Plasmaphysik, Heinrich-Heine-Universität, Düsseldorf, Germany

5 School Physics and Astronomy, The Queen's University, Belfast, UK

${ }^{6}$ LLNL, Livermore, USA

7 CELIA, Université de Bordeaux, Talence, France

8 Department of Physics, University of Nevada, Reno, Nevada, USA

\begin{abstract}
We have performed an experiment aimed at measuring self-generated magnetic fields produced in solids by high electron currents following high-intensity and high contrast short-pulse laser irradiation. This was done using longitudinal high resolution proton deflectometry. The experiment was performed at the Titan-JLF laser facility with a high-power short-pulse beam $(700 \mathrm{fs}, \sim 110 \mathrm{~J})$ split into two beams irradiating two solid targets. One beam is used for the generation of protons and the other beam for the generation of the ultra-high currents of electrons and of the associated magnetic fields. This capability allows us to study the spatio-temporal evolution of the magnetic fields and its dependence on the laser intensity and target material.
\end{abstract}

\section{INTRODUCTION}

The generation and transport of fast electrons produced during the interaction of an ultra-intense, ultra short laser with a solid play an important role in ICF (Inertial Confinement Fusion) within the frame of the fast ignition (FI) scheme, but also in the generation of secondary sources such as X-rays [1] or ions [2]. For all these applications, it is desired that the electrons do not diverge too much, otherwise (1) the efficiency to heat the center of the ICF capsule will be reduced and the FI scheme will fail and (2) the efficiency in producing secondary sources will be low. There have been many studies on ways to prevent such high divergence for the electrons. One possible scheme [3] relies on having strong self-generated toroidal magnetic fields growing within the target and inducing collimation of the electron beam. Such magnetic fields can grow within the solid since the fast electron beam is not neutralized everywhere by the compensating cold return current. The magnetic field arises from the resistive electric field $\eta \mathbf{j}$ which depends on the resistivity $\eta$ and on the return current $\mathbf{j}$. As shown below in Eq. (1), from MaxwellFaraday equation, we can infer that there are in fact two source terms for the magnetic field growing in the solid. One term is a current term (first term on the right-hand side of Eq. (1)) and the other one is a resistive term (second term on the right-hand side of Eq. (1)). This last term depends on the collision

This is an Open Access article distributed under the terms of the Creative Commons Attribution License 2.0, which permits unrestricted use, distribution, and reproduction in any medium, provided the original work is properly cited. 




(a)



(b)

Figure 1. (a) Experimental set-up of the experiment. (b) Focal spot at full energy after plasma mirror.

time, atomic number of the target, temperature, which all evolve in time.

$$
\frac{\partial \mathbf{B}}{\partial t}=-(\eta \nabla \times \mathbf{j}+\nabla \eta \times \mathbf{j}) .
$$

To clarify the importance of such magnetic fields growing within the solids, and to evaluate their ability to indeed collimate electrons, we performed an experiment aimed at measuring them. This was done by performing proton probing [4] of these fields using an auxiliary proton probing beam. As the magnetic fields are toroidal (with respect to the axis of the electrons propagating inside the target, i.e. along the target normal), the probing protons are sent in the target parallel to the target normal, so that the magnetic fields can be measured through the deflections impaired on them by the Lorentz force $\mathbf{v} \times \mathbf{B}$ [4]. To prevent the probing protons to be sensitive mostly to magnetic fields developing on the target surface, e.g. at the target front in the low-density plasma induced by the laser pedestal, we have temporally cleaned the laser producing the fast electrons using a plasma mirror. We also used thin targets as interaction targets to get compensation of B-fields at the front and rear of the target. Here we will mainly present this experimental platform, with some first results being presented and discussed at the end.

\section{EXPERIMENTAL SET-UP}

We performed the experiment using the TITAN facility at LLNL. The use of a split mirror allowed us to have two CPA (Chirped Pulse Amplification) beams, one (CPA 1) as interaction and the other (CPA 2) for the proton radiography diagnostic. Each beam had an energy of approximately $55 \mathrm{~J}$ depending on the shot, for a pulse duration of $700 \mathrm{fs}$. The experimental set-up is shown in Fig. 1(a).

As shown in Fig. 1(a), we used a plasma mirror (PM) before focusing the interaction beam (CPA 1) onto the interaction target in order to have a sharp temporal rising edge for the laser pulse. As mentioned above, our motivation for doing this was to avoid producing a preplasma in front of the interaction target where large magnetic fields due to hydrodynamic expansion of the plasma would grow, impacting the possibility to measure the magnetic fields due to hot electrons current. Since the best fluence for our PM was between 90 and $110 \mathrm{~J} \cdot \mathrm{cm}^{-2}$, it was placed at $2.7 \mathrm{~cm}$ from the target to have a fluence of $100 \mathrm{~J} \cdot \mathrm{cm}^{-2}$. We had after the PM approximately $70 \%$ of the incident energy and a focal spot size between 7 and $8 \mu \mathrm{m}$ (see Fig. 1(b)). Moreover, to maintain similar magnetic fields growing on the front and rear surfaces of the target (as a result of electron streaming from the solid into vacuum [3]), we used thin targets ( $3 \mu \mathrm{m}$ thick).

As mentioned above, in order to measure the self-generated magnetic fields, we used face-on proton radiography (see Fig. 2(a)) through the dense regions of the plasma [4]. For this, as shown in Fig. 1(a), 


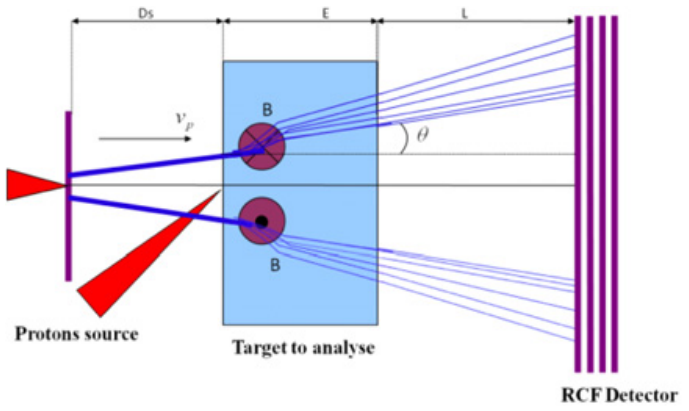

(a)



(b)

Figure 2. (a) Proton radiography principle. (b) Typical spectrum of the probing protons obtained during the experiment.

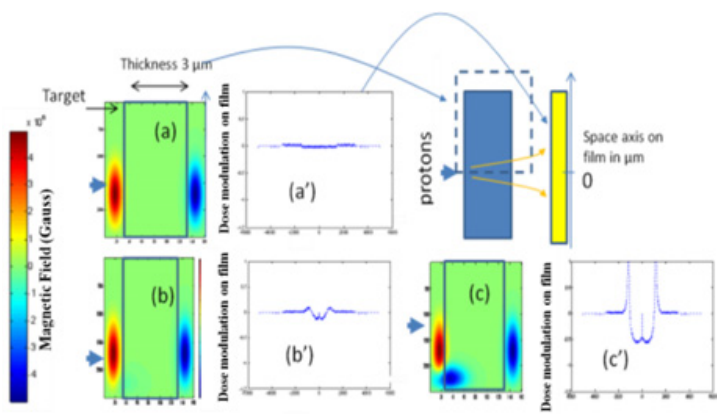

Figure 3. Justification of the ability to probe magnetic fields in dense matter.

CPA 2 interacted with a solid target. By the TNSA (Target Normal Sheath Acceleration) mechanism [5], protons were emitted with a small divergence angle $\left(\sim 20^{\circ}\right)$ and with peak energies between 10 and 30 $\mathrm{MeV}$ (see a typical proton spectrum in Fig. 2(b)). After passing through the interaction target and being deflected by the magnetic fields following Lorentz's law $(q \mathbf{v} \times \mathbf{B})$ the protons were collected by a stack of radiochromic films (RCF). Since protons of different energy have different time of flight between the source foil and the interaction target, we can analyze, in a single shot, the deflections impaired by the magnetic fields at different times during the interaction.

This is possible since protons deposit their dose in different layers of a stack of RCF according to their incident energy in the stack. In practice, we were able to see the magnetic field dynamics over a time span of $\sim 100 \mathrm{ps}$. The magnification of the radiography onto the RCFs (spatial resolution about 6-8 $\mu \mathrm{m}$ ) was in our case approximately 9.

\section{JUSTIFICATION OF THE ABILITY TO PROBE MAGNETIC FIELD INSIDE TARGET}

We used a particle tracing code [6] in order to assess our ability to probe magnetic fields within the dense part of the target despite the presence of fields at the target surface, as illustrated schematically in Fig 1(a). To do so, as illustrated in the right part of Fig. 3, we simulate protons deflections in different configurations where we change the magnetic field topology. Only the upper half, in a 2D plane, of the target is simulated since we assume the situation to be azimuthally symmetric. In all configurations, 


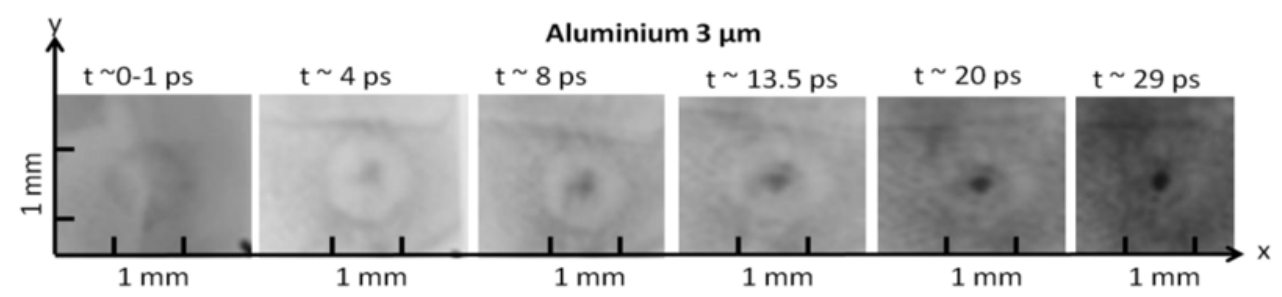

Figure 4. Results of proton radiography through a $3 \mu \mathrm{m}$ thick Al target.

protons are sent through the magnetic field (see Figs. 3(a-c)) and the deviations (induced by the fields) from uniform dose deposition on the RCF are plotted in Figs. 3 (a'-c').

As can be seen in Fig. 3(a'), when the two magnetic fields at the target back and front are of the same amplitude (but of reversed polarity), we do not observe any significant modulation on the detector. The proton deposited dose is uniform, as it would be if there were no fields at all since indeed the deflections impaired by the two opposite fields almost cancel each other out. Now, if we introduce a difference of $20 \%$ between these two fields (see Fig. 3(b)), we can observe a modulation of the proton dose on the film, although it is of a small absolute amplitude. Finally, if we add a third magnetic field inside the target, as can be expected in a more realistic case [7] and as illustrated in Fig. 1(a), we observe a strong modulation, similar to what is seen experimentally (see section 4). Provided that the magnetic fields would indeed be similar on the front and rear surface, this technique would thus indeed enable us to probe magnetic fields within the dense part of the target.

\section{PRELIMINARY RESULTS AND DISCUSSION}

Figure 4 displays typical proton deflection patterns observed on the RCF films obtained in a single shot. Higher energy protons probe the target at earlier times whereas lower energy ones probe it later. Using the code SRIM [8], we can determine the mean energy of the protons having deposited their energy in a particular RCF layer. Knowing the distance between the proton source target and the interaction target, as well as the time delay between CPA 1 and CPA 2, we can determine for a given RCF layer the time at which the protons have probed the interaction target. These times are indicated above each RCF film shown in Fig. 4. Time 0 corresponds to the moment when CPA 1 strikes the interaction target.

As can be seen for the case of an Aluminium interaction target displayed in Fig. 4, at the time of approximately $0-1 \mathrm{ps}$, the observed outward deflection of protons allows us to say that the self-generated magnetic field, at that time, would tend to collimate the electrons propagating within the target since they push out the probing protons. After that, we observe an inversion of the magnetic fields which would correspond to magnetic fields that would make the electrons diverge. We have performed such probing also for other materials $(\mathrm{Au}, \mathrm{Cu}, \mathrm{CH})$; analysis of those is under way and will be reported in a future publication.

\section{CONCLUSION}

We performed a first experiment aimed at probing self-generated magnetic fields linked with fast electron transport in dense targets. Using a plasma mirror and thin targets, we were able to probe self-generated magnetic fields in dense targets. The experiment shows that the dynamics of this selfgenerated magnetic field takes place over a very large time scale, i.e. $\sim 100 \mathrm{ps}$ and is more complex than expected. 
IFSA 2011

\section{References}

[1] B. Dromey et al, Phys. Rev. Lett. 99, 085001 (2007)

[2] J. Fuchs et al, C. R. Physique 10, 176 (2009)

[3] Y. Sentoku et al, Phys. Rev. Lett. 90, 155001 (2003)

[4] C.A Cecchetti et al, Phys. Plasmas 16, 043102 (2009)

[5] S.C Wilks et al, Phys.of Plasmas 8, 542 (2001)

[6] L Lancia et al, Phys. Plasmas 18, 030705 (2011)

[7] Y Sentoku et al, Phys. Rev. Lett. 107, 135005 (2011)

[8] J F Ziegler et al, The stopping and Range of Ions in Solids, Pergamon, New York, 1996 